\title{
DIVERSIDAD Y DISTRIBUCIÓN DE CINCO FAMILIAS DE PAPILIONOIDEA (LEPIDOPTERA) DE LAS SELVAS ALTAS EN LA PROVINCIA BIOGEOGRÁFICA DEL GOLFO DE MÉXICO
}

\author{
DIVERSITY AND DISTRIBUTION OF FIVE FAMILIES OF PAPILIONOIDEA \\ (LEPIDOPTERA) OF THE HIGH EVERGREEN TROPICAL FOREST \\ AT THE MEXICAN GULF BIOGEOGRAPHIC PROVINCE
}

\author{
IGNACIO FLORES-CONTRERAS y MERCEDES LUNA-REYES*
}

\begin{abstract}
Colección Lepidopterológica, Museo de Zoología, Facultad de Estudios Superiores Zaragoza, UNAM. Batalla 5 de mayo s/n, Ejército de Oriente, Iztapalapa, C.P. 09230. Ciudad de México.

* Autor de correspondencia:<mmluna@puma2.zaragoza.unam.mx>; <mercedesluna6@gmail.com>.
\end{abstract}

Recibido: 25/05/2016; aceptado: 17/05/2017

Editor responsable: Pedro Reyes Castillo.

Flores-Contreras, I. y Luna-Reyes, M. (2017). Diversidad y distribución de cinco familias de Papilionoidea (Lepidoptera) de las selvas altas en la provincia biogeográfica del Golfo de México. Acta Zoológica Mexicana (n.s.), 33(2), 211-230.

RESUMEN. Se evaluó la riqueza de las Papilionoidea de las selvas altas en la provincia biogeográfica del Golfo de México, y sus relaciones biogeográficas con base en su distribución en 24 sitios mediante la aplicación del análisis de parsimonia de endemismos (PAE) y del índice de Jaccard. Se encontró un total de 793 especies, 218 de las cuales fueron consideradas Exclusivas de las selvas altas y de importancia para estudios de conservación, y 86 de estas como Restringidas con distribución limitada en este tipo de vegetación. Las 218 especies Exclusivas de las selvas altas fueron utilizadas para obtener dos dendrogramas que permitieron agrupar los sitios estudiados en un conjunto Norte y otro Sur con referencia a la Sierra de Chiconquiaco, agrupación que apoya la idea de dividir a la provincia biogeográfica en dos distritos.

Palabras clave: bosque tropical perennifolio, conservación, dendrograma, índice de Jaccard, mariposas diurnas, PAE, provincia biogeográfica, riqueza específica.

\section{INTRODUCCIÓN}

Las selvas altas perennifolia y subperennifolia (SA) son comunidades vegetales clasificadas dentro del Bosque Tropical Perennifolio (Challenger \& Soberón, 2008), tipo de vegetación considerado de importancia para la conservación, ya que a pesar de cubrir sólo 7\% de la superficie continental, alberga el 50\% de la biodiversidad mundial (Wilson, 1988); además, este tipo de vegetación se encuentra amenazado por las actividades enfocadas a la explotación de sus especies maderables y no maderables,
Flores-Contreras, I., \& Luna-Reyes, M. (2017). Diversity and distribution of five families of Papilionoidea (Lepidoptera) of the high evergreen tropical forest at the Mexican Gulf biogeographic province. Acta Zoológica Mexicana (n.s.), 33(2), 211-230.

ABSTRACT. In this work, we analyzed the richness and biogeographic relations of Papilionoidea at the Mexican Gulf Biogeographic Province. It applied PAE and Jaccard index at 24 sites and we find 793 species was associated at high evergreen tropical forest (ETF), of which 218 were Exclusives species in this vegetation type and they can used in conservation studies, and 86 of these as Restricted with limited distribution in ETF. In this analysis, the studied sites formed two groups, one at north and another at south of the Sierra Chiconquiaco. These groups support the idea of the Mexican Gulf biogeographic province separated in two districts.

Key words: biogeographic province, butterflies, conservation, Jaccard index, PAE, richness, evergreen tropical forest.

así como por el cambio de uso de suelo, que rápidamente reducen su extensión a nivel mundial.

En América, su distribución más septentrional se encuentra en la vertiente del Atlántico Mexicano (las planicies del Golfo de México en altitudes de hasta 1,000 msnm, el sur y este de la Península de Yucatán, y el este de Chiapas donde asciende hasta los 1,500 msnm), aunque hay una extensión importante sobre la vertiente del Pacífico, en la Sierra Madre de Chiapas y las faldas de la Sierra Madre del Sur de Oaxaca y Guerrero (Challenger \& Soberón 2008; CONABIO, 1998, 1999). Además, se 
distribuye en el norte de Guatemala, en los departamentos del Petén, Alta Verapaz e Izabal por debajo de los 1,800 msnm (CCAD, 2015b, d), y en 62\% del territorio de Belice alcanzando hasta los 1,000 msnm en las Montañas Mayas (CCAD, 2015a, c; Cherrington et al., 2010). Sin tomar en cuenta la vertiente del Pacífico Mexicano, estas áreas de distribución se ubican dentro de lo que Morrone (2001) y Morrone et al. (1999, 2002) definen como la provincia biogeográfica del Golfo de México (PBGM).

Esta provincia se ubica en el reino Neotropical en la región del Caribe, y corresponde al Neotrópico Húmedo Mesoamericano (Espinosa et al., 2008). Se extiende a lo largo de la costa del Golfo de México, desde la cuenca del Río San Fernando en el estado de Tamaulipas, hacia el sur pasando por los estados de San Luis Potosí, Veracruz, Puebla, Hidalgo y Tabasco, por debajo de los 1,500 msnm, y parte de Oaxaca y Chiapas por debajo de los 1,000 msnm, hasta llegar al Río Candelaria en Campeche, incluso abarca el sur de la región de Calakmul donde inicia la Península de Yucatán (Espinosa et al., 2008); continúa por el norte de Guatemala alcanzando los 1,600 msnm en la Sierra Madre, e incluye la totalidad de Belice (Fig. 1).

La obra base para el estudio de los Papilionoidea en México y Centroamérica, es la Biologia Centrali Americana, elaborada por Godman \& Salvin entre 1879 y 1901. En ella se citan 418 especies en 22 localidades de Veracruz, México (Luis-Martínez et al., 1995); 460 especies en 128 localidades de Guatemala (Salinas-Gutiérrez et al., 2009), y 90 especies en seis localidades de Belice, referido como Honduras Británica (Godman \& Salvin, 1879-1901); estas pueden considerarse las primeras especies reportadas para la PBGM, y en su mayoría para las SA de la provincia.

De acuerdo con Michán et al. (2005) y Luis-Martínez et al. (2011), posterior a Godman y Salvin para esta provincia destacan colecciones como las de Escalante, depositada en el Museo de Zoología de la Facultad de Ciencias, UNAM y que cuenta con 5,000 ejemplares procedentes principalmente de la región de Los Tuxtlas, Veracruz (Luis-Martínez et al., 1995). Trabajos más recientes sobre los Papilionoidea de las SA en la PBGM son

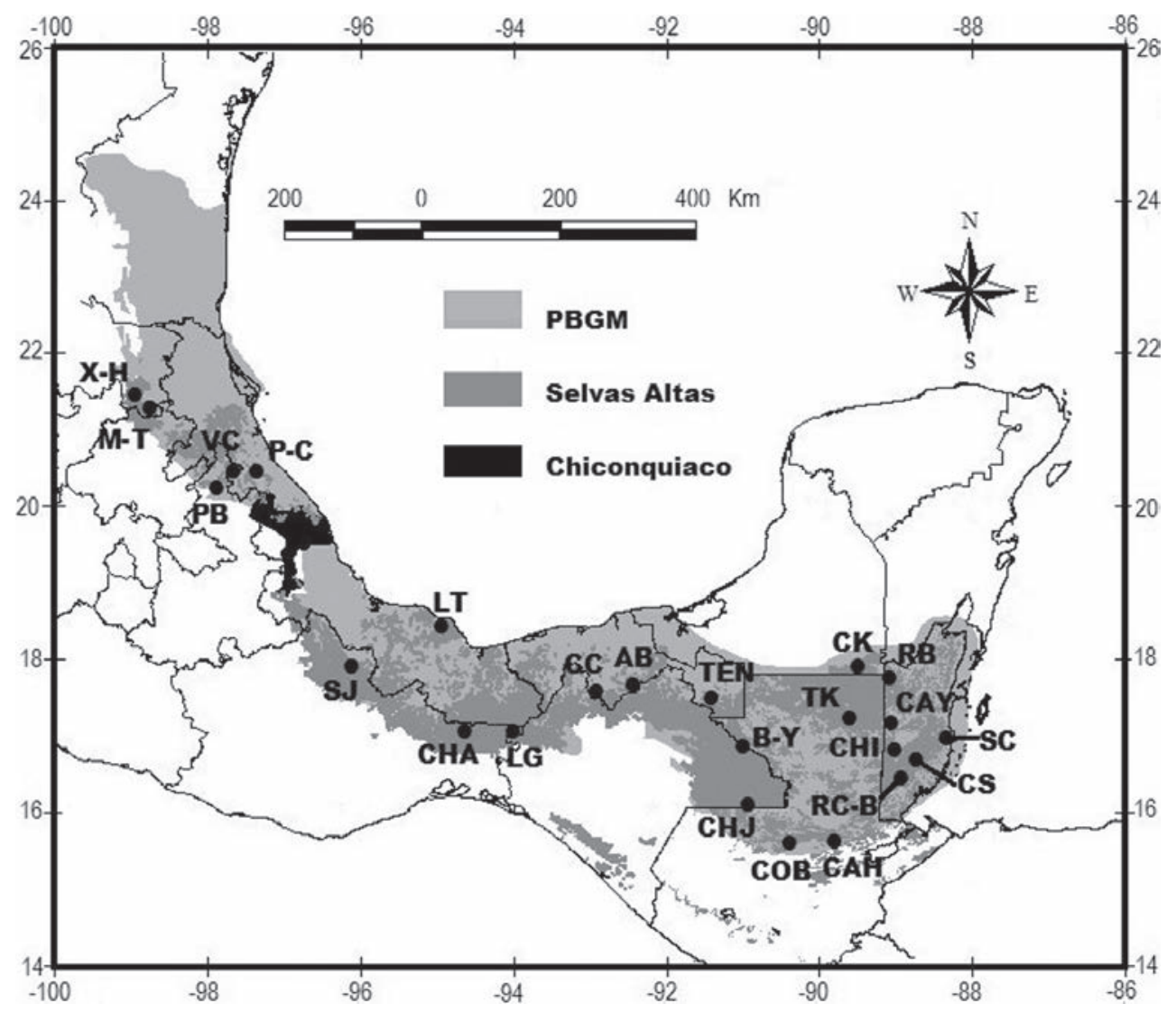

Figura 1. Ubicación de la Sierra de Chiconquiaco y los sitios de selvas altas en la provincia biogeográfica del Golfo de México (PBGM). 
los realizados por de la Maza \& de la Maza (1985a, b), González-Martínez (1996), Luis-Martínez et al. (1991), Martínez (1994), Maya-Martínez et al. (2005), Pozo et al. (2003), Raguso \& Llorente-Bousquets (1990), SalinasGutiérrez et al. (2004, 2006), Salinas-Gutiérrez (2010) y Villegas (1998); sus investigaciones abarcan desde listas regionales de Papilionoidea, su fenología, distribución altitudinal y por tipos de vegetación, hasta comparaciones de la riqueza y posibles relaciones ecológicas y biogeográficas entre localidades del sureste mexicano y Centroamérica.

Después de la publicación de la Biologia Centrali Americana, el trabajo en Guatemala es escaso y se encuentra dirigido principalmente a revisiones y compilaciones del país (Salinas-Gutiérrez et al., 2009). Sin embargo, destacan los trabajos de Welling $(1973,1975,1977)$, colector comercial en bosque mesófilo y SA, el de Austin et al. (1996) en Tikal, el de Barrios et al. (2007) en Alta Verapaz, y el de Salinas-Gutiérrez et al. (2009), quienes realizaron una síntesis de las mariposas del país basada en revisiones de literatura y colecciones científicas.

En Belice los estudios se vieron limitados a las colecciones particulares como la de Gibbs $(1912,1914)$ quien publicó dos artículos de las mariposas de Belice y Guatemala, y Meerman (1999) quien publicó el catálogo de mariposas de Belice a partir de datos de museos y literatura. Actualmente la información derivada de trabajos como estos se resguarda en bases de datos contenidas en el Biodiversity and Environmental Resource Data System of Belize (BERDS) creado en 2005 (Meerman \& Clabaugh, 2012), que permite su uso en cuestiones de estudio, aprovechamiento y conservación.

El conocimiento detallado del número y tipo de especies presentes en un área geográfica determinada es fundamental en diversos aspectos de la conservación biológica. Con el objeto de poder diseñar políticas adecuadas de estudio, uso y protección de los recursos bióticos, en los últimos años ha crecido notablemente la necesidad de contar con inventarios, que deben presentar disponibilidad, precisión y amplitud, si se espera su uso en estas tareas (Cervantes et al., 1994). Sin embargo, la mayoría de los trabajos sobre Papilionoidea de las SA en la PBGM, muestran estudios de áreas puntuales que no consideran la composición total de especies en estas comunidades vegetales de la provincia, y sólo algunos están enfocados a comparar la composición de Papilionoidea entre distintos tipos de vegetación o analizar su distribución para determinar áreas de estudio y conservación de las SA. De hecho, salvo los trabajos elaborados por Maya-Martínez et al. (2005), Salinas-Gutiérrez (2010) y Salinas-Gutiérrez et al. $(2004,2006)$ ninguno ha intentado analizar en conjunto la comunidad de Papilionoidea de las selvas del sureste mexicano, la región Huasteca y Centroamérica; y menos aún su relación histórica. Por ello, el presente trabajo muestra un análisis general de la riqueza de cinco de las siete familias de Papilionoidea (Nieukerken et al., 2011) de las SA en la PBGM y de las relaciones existentes entre los sitios estudiados dentro de la provincia, la cual corresponde a una unidad natural de estudio que abarca la mayor parte del área de distribución de esta vegetación en México, Guatemala y Belice, y representa su límite de distribución boreal en América.

\section{MATERIALES Y MÉTODOS}

Sitios y taxones. En este trabajo se utilizó la información de los Papilionoidea proveniente de las publicaciones de Austin et al. (1996), Barrios et al. (2007), GonzálezMartínez (1996), González-Valdivia et al. (2016), Hernández-Baz et al. (2016), Luis-Martínez et al. (1991), Maya-Martínez et al. (2005), Raguso \& Llorente (1997), Raguso \& Llorente-Bousquets (1990), Salinas-Gutiérrez (2010), Salinas-Gutiérrez et al. (2004, 2006), las cuales proporcionan información obtenida de recolectas y de la revisión de bases de datos y literatura, por lo que se consideran los trabajos más completos para las áreas que representan; además, se incluyeron datos procedentes de la megabase MARIPOSA (Luis-Martínez et al., 2005) para San Luis Potosí, la Colección Nacional de Insectos Lepidoptera (CNINLEPIDOPTERA, 2010) del Instituto de Biología (UNAM), el BERDS (Meerman \& Clabaugh, 2012), y de la Colección Lepidopterológica del Museo de Zoología de la FES Zaragoza (UNAM). Con base en los datos recabados se reconocieron 24 sitios ubicados en vegetación de SA de la PBGM (Fig. 1): Xilitla-Huichihuayán (X-H), Matlapa-Tamazunchale (M-T), Barranca de Patla (PB), Venustiano Carranza (VC), Papantla-Coatzintla (PC), Los Tuxtlas (LT), Sierra de Juárez (SJ), Chalchijapa (CHA), La Gringa (LG), Cerro Cocona (CC), Agua Blanca (AB), Tenosique (TEN), Bonampak-Yaxchilán (B-Y), Chajul (CHJ) y Calakmul (CK) para México; Tikal (TK), Cobán (COB) y Cahabón (CAH) para Guatemala; y Río Bravo (RB), Cayo (CAY), Chiquibul (CHI), Stann Creek (SC), Cockscomb (CS) y Río Columbia-Bladen (RC-B) para Belice. A partir de los papilionoideos presentes en cada sitio se elaboró la lista de las especies asociadas a las SA de la PBGM y con otras vegetaciones de las cin- 
co familias consideradas en este análisis (Papilionidae, Pieridae, Lycaenidae, Riodinidae y Nymphalidae), para definir las especies más afines o exclusivas a las SA en esta provincia. La información se registró en una hoja de cálculo de Excel (Microsoft, 2013); los nombres y la distribución de las especies se confirmaron con base en Llorente-Bousquets et al. (2006), Meerman (1999), Salinas-Gutiérrez et al. (2009) y Warren et al. (2013). Posteriormente se comparó el número total de especies reportadas en los países que conforman la PBGM, con el número de especies que presenta cada uno en las SA de la provincia.

Como parte del análisis de la riqueza, las especies se separaron en dos categorías según su incidencia en otros tipos de vegetación dentro de la provincia: 1) Exclusivas a las SA: especies registradas únicamente en las SA dentro de la PBGM, las cuales fueron agrupadas en Restringidas, si se registraron sólo en un sitio; o No Restringidas si se registraron en dos o más sitios. 2) Presentes en otros tipos de vegetación: especies registradas en SA que además han sido recolectadas en Selva Mediana, Selva Baja Caducifolia, Bosque Mesófilo, Bosque de Pino, Bosque de Encino, Manglar, y áreas de Agricultura y pastizal inducido dentro de la PBGM, agrupadas como especies de Presencia moderada si se registraron en la SA y un tipo de vegetación adicional, o generalistas si fueron recolectadas en SA y en dos o más tipos de vegetación distintos.

Similitud y Análisis de parsimonia de endemismos. El análisis de similitud permite establecer la relación entre distintas áreas mediante la comparación de los conjuntos de especies que presentan. Se utilizó el índice de Jaccard para obtener los valores de similitud entre los sitios estudiados (Jaccard, 1908; Koleff, 2005; Magurran, 1988; Moreno, 2001; Southwood \& Henderson, 2000), descrito como $\mathrm{Ij}=\mathrm{c} /(\mathrm{a}+\mathrm{b}-\mathrm{c})$. Este índice se basa en datos de incidencia y no toma en cuenta las ausencias compartidas, ya que considera que comparar la falta de una especie en dos áreas distintas no aporta información relevante. Con estos valores de similitud se construyeron agrupamientos por el método UPGMA (Unweighted pair-group method using arithmetic averages); tanto el Índice de Jaccard como el UPGMA fueron aplicados mediante el programa NTSYSpc 2.11T (Rohlf, 2004).

El análisis de parsimonia de endemismos o PAE (Parsimony Analysis of Endemicity), consiste en la obtención de un cladograma de áreas a partir de las especies compartidas por éstas. Cada clado formado representa áreas con taxones comunes y permite suponer una relación históri- ca, ecológica o geológica (Escalante \& Morrone, 2003; Morrone, 1994a, b, 2004, 2014). En el PAE se empleó el programa Winclada 1.00.08 (Nixon, 1999) mediante una búsqueda heurística con el procedimiento de bisección y reconexión múltiple (Multiple TBR+TBR).

Para la aplicación del índice de Jaccard y el PAE se elaboraron matrices de incidencia de los papilionoideos que únicamente fueron registrados en la SA.

\section{RESULTADOS}

Sitios y taxones. Se obtuvo una lista (no se presenta en este trabajo) de 793 especies pertenecientes a cinco de las siete familias que conforman a Papilionoidea (Niukerken et al., 2011) para las SA de la PBGM (348 Nymphalidae, 192 Lycaenidae, 145 Riodinidae, 65 Pieridae, 43 Papilionidae). El Cuadro 1 muestra que México tiene el mayor número de especies registradas en las SA de la PBGM (709), seguido por Belice (422) y Guatemala (405); las subfamilias con mayor riqueza son Theclinae (182) y Riodininae (133). Los 24 sitios definidos a partir de las fuentes consultadas tuvieron entre 517 y 71 especies: LT (517), SJ (365), CHJ (364), X-H (332), CAY (329), PB (318), TIK (308), M-T (270), B-Y (252), RB (234), CC (210), CHI (199), COB (181), CHA (176), CK (175), TEN (160), LG (159), SC (158), RC-B (151), P-C (137), AB (129), VC (125), CAH (90) y CS (71).

De acuerdo con las especies registradas en cada país se encontró que Belice cuenta con el mayor porcentaje (77.43\%) presente en las SA (Cuadro 2). De las 793 especies de Papilionoidea reportadas para SA en México, Guatemala y Belice, sólo 288 son comunes y 76 (de México y Belice) probablemente comunes a los tres países, lo que da un total de 364, equivalentes al $45.84 \%$ del total reportado para las SA de la PBGM (Cuadro 3). Los valores totales del número de especies se tomaron de LlorenteBousquets et al. (2006) para México, Salinas-Gutiérrez et al. (2012) para Guatemala y Salinas-Gutiérrez (2010) para Belice. Se encontró una diferencia de 14 especies al comparar el total de Papilionoidea de las SA en la PBGM (793) obtenido en este trabajo, con el total de especies (779) referido para la Selva Maya por Salinas-Gutiérrez (2010), aunque al final 46 de los 793 papilionoideos de las SA en la PBGM no fueron mencionados en el trabajo de la Selva Maya (Cuadro 4).

Con respecto a su categoría de incidencia, de los 793 papilionoideos se reconocieron 218 especies exclusivas a las SA (Cuadro 5), 86 de ellas como Restringidas y 132 
Cuadro 1. Riqueza por familia y subfamilia de Papilionoidea de las selvas altas (SA) para cada país que integra la provincia biogeográfica del Golfo de México (PBGM). M: México; G: Guatemala; B: Belice.

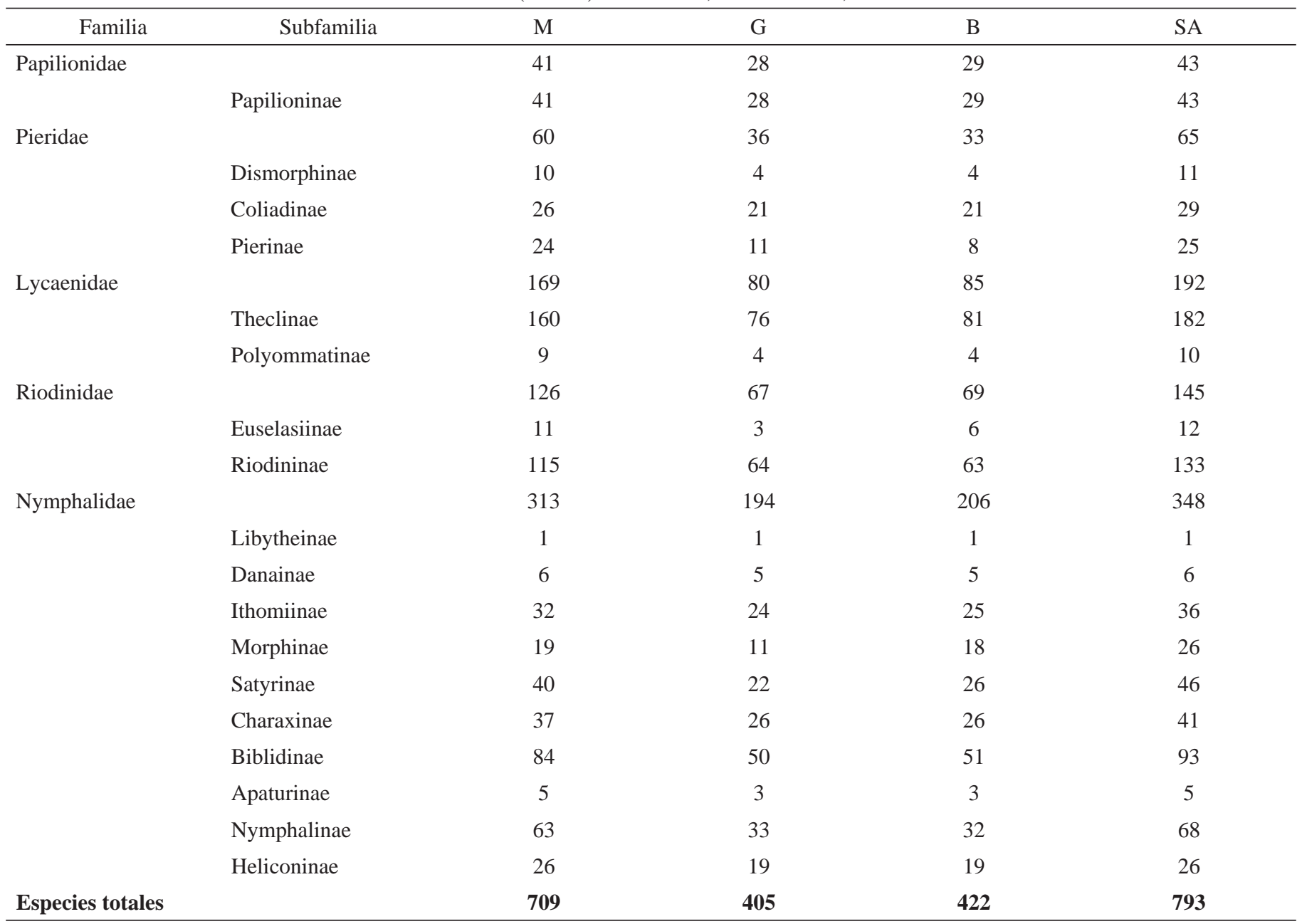

Fuente: Austin et al. (1996), Barrios et al. (2007), González-Martínez (1996), González-Valdivia et al. (2016), Hernández-Baz et al. (2016), Luis-Martínez et al. (1991), Maya-Martínez et al. (2005), Raguso \& Llorente (1997), Raguso \& Llorente-Bousquets (1990), Salinas-Gutiérrez (2010) y de Salinas-Gutiérrez et al. (2004, 2006), así como la base de datos de BERDS (Meerman \& Clabaugh, 2012) y de las colecciones de mariposas del Museo de Zoología de la Facultad de Ciencias (Luis-Martínez et al., 2005), del Instituto de Biología (CNINLEPIDOPTERA, 2010), y del Museo de Zoología de la FES Zaragoza, todas de la UNAM.

Cuadro 2. Total de Papilionoidea de México (M), Guatemala (G) y Belice (B), con el número y proporción de especies (\%) presentes en las SA de la PBGM.

\begin{tabular}{lcccc}
\hline \multirow{2}{*}{ Familia } & \multicolumn{4}{c}{ Especies totales/Especies en la SA } \\
\cline { 2 - 5 } & SA & M & Gt & B \\
\hline Papilionidae & $79 / 41$ & $40 / 28$ & $36 / 29$ & 43 \\
Pieridae & $109 / 60$ & $69 / 36$ & $40 / 33$ & 65 \\
Lycaenidae & $255 / 169$ & $162 / 80$ & $140 / 85$ & 192 \\
Riodinidae & $203 / 126$ & $120 / 67$ & $99 / 69$ & 145 \\
Nymphalidae & $544 / 313$ & $358 / 194$ & $230 / 206$ & 348 \\
Total & $1190 / 709$ & $749 / 405$ & $545 / 422$ & 793 \\
$\%$ & 59.57 & 54.07 & 77.43 & \\
\hline
\end{tabular}

como No Restringidas, además de 575 especies que también se distribuyeron en otros tipos de vegetación, 166 de Presencia moderada y 409 Generalistas.

Similitud y Análisis de parsimonia de endemismos. En los análisis de similitud y PAE se utilizaron las 218 especies de Papilionoidea exclusivas de las SA; de esta manera, sólo se consideraron las especies que tienen una relación más estrecha con esta vegetación y se omitieron aquellas cuya relación o dependencia es menor o incierta. Del primer análisis se obtuvo el dendrograma de similitud (Fig. 2a) donde se observó la formación de dos grupos principales: uno conformado por los sitios ubicados al norte de la Sierra de Chiconquiaco: X-H, M-T, VC, P-C y $\mathrm{CK}$ (aunque éste último geográficamente se encuentra en 
Flores-Contreras y Luna-Reyes: Papilionoidea de las selvas altas en el Golfo de México

Cuadro 3. Papilionoidea de las SA de la PBGM compartidas entre México (M), Guatemala (G) y Belice (B). Porcentaje respecto al total de especies de las SA (\%).

\begin{tabular}{|c|c|c|c|c|c|c|c|c|c|}
\hline Familia & Subfamilia & M-G & M-B & G-B & M-G-B & $\mathrm{M}$ & G & B & SA \\
\hline \multirow[t]{2}{*}{ Papilionidae } & & 3 & 4 & 0 & 24 & 10 & 1 & 1 & 43 \\
\hline & Papilioninae & 3 & 4 & 0 & 24 & 10 & 1 & 1 & 43 \\
\hline \multirow[t]{4}{*}{ Pieridae } & & 7 & 8 & 1 & 24 & 21 & 4 & 0 & 65 \\
\hline & Dismorphinae & 0 & 1 & 0 & 3 & 6 & 1 & 0 & 11 \\
\hline & Coliadinae & 3 & 5 & 1 & 15 & 3 & 2 & 0 & 29 \\
\hline & Pierinae & 4 & 2 & 0 & 6 & 12 & 1 & 0 & 25 \\
\hline \multirow[t]{3}{*}{ Lycaenidae } & & 21 & 22 & 3 & 48 & 78 & 8 & 12 & 192 \\
\hline & Theclinae & 21 & 22 & 2 & 45 & 72 & 8 & 12 & 182 \\
\hline & Polyommatinae & 0 & 0 & 1 & 3 & 6 & 0 & 0 & 10 \\
\hline \multirow[t]{3}{*}{ Riodinidae } & & 16 & 17 & 6 & 39 & 54 & 6 & 7 & 145 \\
\hline & Euselasiinae & 1 & 3 & 0 & 2 & 5 & 0 & 1 & 12 \\
\hline & Riodininae & 15 & 14 & 6 & 37 & 49 & 6 & 6 & 133 \\
\hline \multirow[t]{11}{*}{ Nymphalidae } & & 27 & 25 & 7 & 153 & 108 & 7 & 21 & 348 \\
\hline & Libytheinae & 0 & 0 & 0 & 1 & 0 & 0 & 0 & 1 \\
\hline & Danainae & 0 & 0 & 0 & 5 & 1 & 0 & 0 & 6 \\
\hline & Ithomiinae & 3 & 5 & 1 & 18 & 6 & 2 & 1 & 36 \\
\hline & Morphinae & 0 & 3 & 1 & 9 & 7 & 1 & 5 & 26 \\
\hline & Satyrinae & 3 & 1 & 0 & 19 & 17 & 0 & 6 & 46 \\
\hline & Charaxinae & 5 & 1 & 0 & 21 & 10 & 0 & 4 & 41 \\
\hline & Biblidinae & 8 & 9 & 5 & 35 & 32 & 2 & 2 & 93 \\
\hline & Apaturinae & 0 & 0 & 0 & 3 & 2 & 0 & 0 & 5 \\
\hline & Nymphalinae & 6 & 4 & 0 & 25 & 28 & 2 & 3 & 68 \\
\hline & Heliconinae & 2 & 2 & 0 & 17 & 5 & 0 & 0 & 26 \\
\hline Total & & 74 & 76 & 17 & 288 & 271 & 26 & 41 & 793 \\
\hline$\%$ & & 9.31 & 9.57 & 2.14 & 36.27 & 34.17 & 3.27 & 5.16 & 100 \\
\hline
\end{tabular}

el sur), y otro integrado por aquellos ubicados al sur (LT, SJ, B-Y, CHJ, TK, CAY, RB, SC, RC-B, CHI, CS, COB, CAH, CHA, CC, LG, AB y TEN). Con el PAE (Fig. 2b) se generó un árbol no resuelto en su totalidad (consenso de mayoría $\mathrm{L}=456, \mathrm{CI}=48$ y $\mathrm{RI}=31$, a partir de 14 árboles con $\mathrm{L}=428, \mathrm{CI}=51$ y $\mathrm{RI}=39$ ), que presentó una agrupación similar a la del grupo norte observado en el dendrograma de similitud, excepto que CK es excluido del clado que agrupa a los sitios del norte; en ambos árboles PB se mantiene separado del resto de los sitios.

Se revisó la lista de papilionoideos para distinguir las especies que definieron las agrupaciones obtenidas en los análisis de similitud y PAE, y se encontró que de las 218 especies exclusivas de las SA, 17 fueron reportadas a ambos lados de la sierra de Chiconquiaco, ocho sólo en el norte y 193 únicamente en el sur; Finalmente, 411 del total de especies (793) están presentes tanto al norte como al sur de Chiconquiaco, otras 39 sólo se registraron al norte y 343 exclusivamente en el sur.

\section{DISCUSIÓN}

Sitios y taxones. La lista de papilionoideos para las SA de la PBGM se integró por 793 especies pertenecientes a cinco de las siete familias que conforman a Papilionoidea (Niukerken et al., 2011), con proporciones de riqueza que de manera general coinciden con los reportados en la literatura para la superfamilia a cualquier escala espacial que se estudie la región neotropical (Lamas, 2008; Llorente-Bousquets et al., 2014; Luis-Martínez et al., 2004), excepto en los casos donde existen ambientes fuertemente alterados, sitios con muestreo escaso o inadecuado, o muestreos parciales en sitios con estacionalidad muy mar- 
Cuadro 4. Papilionoideos de las SA en la PBGM adicionales a la lista de la Selva Maya (Salinas-Gutiérrez 2010); los nombres completos de los sitios se indican en el texto.

\begin{tabular}{|c|c|c|c|}
\hline Familia & Subfamilia & Especie & Sitios \\
\hline \multirow[t]{2}{*}{ Papilonidae } & Papilioninae & Pterourus multicaudata multicaudata (W.F. Kirby, 1884) & $\mathrm{PB}$ \\
\hline & & Pterourus pilumnus (Boisduval, 1836) & $\mathrm{X}-\mathrm{H}, \mathrm{M}-\mathrm{T}, \mathrm{PB}$ \\
\hline \multirow{5}{*}{ Pieridae } & Coliadinae & Colias eurytheme Boisduval, 1852 & PB \\
\hline & Pierinae & Hesperocharis crocea crocea H.W. Bates, 1866 & $\mathrm{~PB}$ \\
\hline & & Catasticta teutila teutila (Doubleday, 1847) & PB \\
\hline & & Pieris rapae rapae (Linnaeus, 1758) & LT \\
\hline & & Perrhybris pamela mapa J. Maza \& R.G. Maza, 1989 & $\mathrm{~B}-\mathrm{Y}, \mathrm{CHJ}$ \\
\hline \multirow[t]{8}{*}{ Lycaenidae } & Theclinae & Temecla paron (Godman \& Salvin, 1887) & $\mathrm{PB}$ \\
\hline & & Contrafacia bassania (Hewitson, 1868) & $\mathrm{X}-\mathrm{H}$ \\
\hline & & Ocaria clenchi (K. Johnson, 1992) & M-T, TK \\
\hline & & Calycopis tamos (Godman \& Salvin, 1887) & RC-B \\
\hline & & Ministrymon cleon (Fabricius, 1775) & $\mathrm{X}-\mathrm{H}$ \\
\hline & & Iaspis sp. Robbins, MS & LT, CC \\
\hline & & Erora subflorens (Schaus, 1913) & $\mathrm{PB}, \mathrm{LT}$ \\
\hline & Polyommatinae & Hemiargus hanno antibubastus Hübner, [1818] & X-H, P-C, LT, CHA, LG, SJ, CC, B-Y \\
\hline \multirow[t]{5}{*}{ Riodinidae } & Riodininae & Pheles strigosa strigosa (Staudinger, 1876) & CHJ \\
\hline & & Calephelis perditalis donahuei McAlpine, 1971 & $\mathrm{M}-\mathrm{T}$ \\
\hline & & Calephelis rawsoni McAlpine, 1939 & $\mathrm{P}-\mathrm{C}$ \\
\hline & & Melanis cephise acroleuca (R. Felder, 1869) & $\mathrm{LT}$ \\
\hline & & Menander pretus picta (Godman \& Salvin, 1886) & $\mathrm{RC}-\mathrm{B}$ \\
\hline \multirow{15}{*}{ Nymphalidae } & Satyrinae & Cissia undina (Butler, 1870) & $\mathrm{X}-\mathrm{H}, \mathrm{LT}, \mathrm{CC}$ \\
\hline & & Euptychia fetna Butler, 1870 & $\mathrm{LT}$ \\
\hline & Charaxinae & Hypna clytemnestra mexicana A. Hall, 1917 & $\mathrm{LT}$ \\
\hline & & Prepona deiphile diaziana L.D. Miller \& J.Y. Miller, 1976 & $\mathrm{CHI}$ \\
\hline & Biblidinae & Myscelia cyaniris alvaradia R.G. Maza \& Díaz, 1982 & $\mathrm{P}-\mathrm{C}$ \\
\hline & & Nica flavilla canthara (Doubleday, 1849) & TK, COB, RC-B, CHI, CS \\
\hline & & Adelpha erymanthis esperanza Balcázar \& Willmott, 2003 & $\mathrm{RC}-\mathrm{B}, \mathrm{CHI}$ \\
\hline & & Adelpha malea fundania Fruhstorfer, 1915 & LT, SJ, CC, CHJ \\
\hline & Nymphalinae & Hypanartia trimaculata autumna Willmott, J. Hall y Lamas, 2001 & $\mathrm{X}-\mathrm{H}, \mathrm{LT}$ \\
\hline & & Polygonia g-argenteum (Doubleday, 1848) & $\mathrm{PB}$ \\
\hline & & Anthanassa nebulosa alexon (Godman \& Salvin, 1889) & $\mathrm{X}-\mathrm{H}, \mathrm{M}-\mathrm{T}, \mathrm{P}-\mathrm{C}$ \\
\hline & & Anthanassa otanes cyno (Godman \& Salvin, 1889) & $\mathrm{PB}$ \\
\hline & & Phyciodes mylitta thebais Godman \& Salvin, 1878 & $\mathrm{P}-\mathrm{C}, \mathrm{TK}$ \\
\hline & & Phyciodes pallescens (R. Felder, 1869) & $\mathrm{M}-\mathrm{T}, \mathrm{P}-\mathrm{C}$ \\
\hline & & Phyciodes pulchella pulchella (Boisduval, 1852) & $\mathrm{P}-\mathrm{C}$ \\
\hline
\end{tabular}


Cuadro 5. Relación de mariposas exclusivas a la SA que se proponen en este trabajo para el estudio y conservación de este tipo de vegetación.

\begin{tabular}{|c|c|c|c|c|}
\hline \multirow[t]{2}{*}{ No. } & \multirow[t]{2}{*}{ PAPILIONOIDEA } & \multicolumn{3}{|c|}{ especies exclusivas } \\
\hline & & México & Guatemala & Belice \\
\hline & PAPILIONIDAE & & & \\
\hline 1 & Eurytides salvini (H.W. Bates, 1864) & 1 & 1 & 1 \\
\hline 2 & Protesilaus macrosilaus macrosilaus (Gray, [1853]) & & 1 & \\
\hline 5 & Parides panares lycimenes (Boisduval, 1870) & 1 & 1 & 1 \\
\hline \multirow[t]{3}{*}{6} & Heraclides rogeri rogeri (Boisduval, 1836) & 1 & & 1 \\
\hline & PIERIDAE & & & \\
\hline & Dismorphinae & & & \\
\hline 9 & Aphrissa boisduvalii (C. Felder \& R. Felder, 1861) & 1 & & 1 \\
\hline 10 & Pyrisitia nise tenella (Boisduval, 1836) & & 1 & \\
\hline 11 & Eurema agave millerorum Llorente \& Luis, 1987 & 1 & & \\
\hline 12 & Eurema daira lydia (C. Felder \& R. Felder, 1861) & & 1 & 1 \\
\hline \multirow[t]{2}{*}{13} & Eurema fabiola (C. Felder \& R. Felder, 1861) & & 1 & \\
\hline & Pierinae & & & \\
\hline 14 & Hesperocharis costaricensis costaricensis H.W. Bates, 1866 & & 1 & \\
\hline 15 & Catasticta nimbice ochracea (H.W. Bates, 1864) & 1 & & \\
\hline 16 & Melete polyhymnia florinda (Butler, 1875) & 1 & 1 & \\
\hline 20 & Paiwarria antinous (C. Felder \& R. Felder, 1865) & 1 & & 1 \\
\hline 21 & Brangas caranus (Stoll, 1780) & & & 1 \\
\hline 22 & Brangas coccineifrons (Godman \& Salvin, 1887) & 1 & 1 & \\
\hline 23 & Enos falerina (Hewitson, 1867) & & 1 & 1 \\
\hline 24 & Atlides halesus (Cramer, 1777) & 1 & & \\
\hline 25 & Theritas hemon (Cramer, 1775) & 1 & & 1 \\
\hline 26 & Theritas theocritus (Fabricius, 1793) & 1 & 1 & \\
\hline 27 & Temecla heraclides (Godman \& Salvin, 1887) & 1 & & \\
\hline 28 & Thereus lausus (Cramer, 1779) & & 1 & \\
\hline 29 & Arawacus aetolus (Sulzer, 1776) & & & 1 \\
\hline 30 & Arawacus leucogyna (C. Felder \& R. Felder, 1865) & & & 1 \\
\hline 31 & Arawacus togarna (Hewitson, 1867) & 1 & & 1 \\
\hline 32 & Contrafacia imma (Prittwitz, 1865) & 1 & 1 & \\
\hline 33 & Kolana lyde (Godman \& Salvin, 1887) & 1 & 1 & 1 \\
\hline
\end{tabular}


Cuadro 5. Continuación.

\begin{tabular}{|c|c|c|c|c|}
\hline No. & PAPILIONOIDEA & & ecies exclusi & \\
\hline & & México & Guatemala & Belice \\
\hline 34 & Ocaria clenchi (K. Johnson, 1992) & 1 & 1 & \\
\hline 36 & Megathecla cupentus (Stoll, 1781) & 1 & & \\
\hline 37 & Janthecla janthina (Hewitson, 1867) & 1 & 1 & 1 \\
\hline 40 & Lamprospilus coelicolor (Butler \& H. Druce, 1872) & & & 1 \\
\hline 41 & Arumecla galliena (Hewitson, 1877) & 1 & 1 & \\
\hline 42 & Camissecla vespasianus (Butler \& H. Druce, 1872) & 1 & & 1 \\
\hline 43 & Ziegleria hoffmani K. Johnson, 1993 & 1 & & \\
\hline 47 & Calycopis atnius (Herrich-Schäffer, 1853) & 1 & & \\
\hline 48 & Calycopis caesaries (H. H. Druce, 1907) & & 1 & \\
\hline 49 & Calycopis cerata (Hewitson, 1877) & & 1 & 1 \\
\hline 50 & Calycopis pisis (Godman \& Salvin, 1887) & & & 1 \\
\hline 51 & Calycopis xeneta (Hewitson, 1877) & & & 1 \\
\hline 52 & Strymon alea (Godman \& Salvin, 1887) & 1 & & \\
\hline 53 & Strymon mulucha (Hewitson, 1867) & 1 & 1 & 1 \\
\hline 54 & Strymon astiocha (Prittwitz, 1865) & 1 & & \\
\hline 61 & Mithras orobia (Hewitson, 1867) & & 1 & \\
\hline 62 & Gargina gargophia (Hewitson, 1877) & 1 & & \\
\hline 63 & Gargina caninius (H.H. Druce, 1907) & 1 & & \\
\hline 64 & Theclopsis leos (Schaus, 1913) & & & 1 \\
\hline 65 & Ostrinotes empusa (Hewitson, 1867) & & 1 & \\
\hline 66 & Strephonota sphinx (Fabricius, 1775) & & & 1 \\
\hline 67 & Strephonota syedra (Hewitson, 1867) & 1 & 1 & 1 \\
\hline 68 & Strephonota ambrax (Westwood, 1852) & 1 & 1 & \\
\hline 69 & Thepytus echelta (Hewitson, 1867) & 1 & & \\
\hline 70 & Oenomaus atesa (Hewitson, 1867) & 1 & & 1 \\
\hline 71 & Michaelus thordesa (Hewitson, 1867) & 1 & & \\
\hline 72 & Ignata n. sp. Robbins, MS & 1 & & \\
\hline 73 & Ignata norax (Godman \& Salvin, 1887) & 1 & & \\
\hline 74 & Hypostrymon critola (Hewitson, 1874) & 1 & & \\
\hline
\end{tabular}


Cuadro 5. Continuación.

\begin{tabular}{|c|c|c|c|c|}
\hline \multirow[t]{2}{*}{ No. } & \multirow[t]{2}{*}{ PAPILIONOIDEA } & \multicolumn{3}{|c|}{ especies exclusivas } \\
\hline & & México & Guatemala & Belice \\
\hline 75 & Nesiostrymon dodava (Hewitson, 1877) & 1 & & \\
\hline 77 & Iaspis n. sp. Robbins, MS & 1 & & \\
\hline 78 & Iaspis temesa (Hewitson, 1868) & 1 & & \\
\hline 80 & Polyommatinae & & & \\
\hline 81 & Hemiargus hanno astenidas (Lucas, 1857) & & 1 & 1 \\
\hline \multirow[t]{2}{*}{82} & Echinargus huntingtoni hannoides Clench, 1965 & 1 & & \\
\hline & RIODINIDAE & & & \\
\hline 85 & Euselasia inconspicua (Godman \& Salvin, 1878) & 1 & & \\
\hline \multirow[t]{2}{*}{86} & Euselasia mys mys (Herrich-Schäffer, [1853]) & & & 1 \\
\hline & Riodininae & & & \\
\hline 87 & Perophthalma lasus Westwood, [1851] & 1 & 1 & 1 \\
\hline 88 & Perophthalma tullius (Fabricius, 1787) & & 1 & \\
\hline 89 & Hermathena oweni Schaus, 1913 & 1 & & \\
\hline 90 & Napaea eucharila picina Stichel, 1910 & 1 & 1 & 1 \\
\hline 91 & Cremna actoris (Cramer, 1776) & 1 & & \\
\hline 92 & Cremna thasus subrutila Stichel, 1910 & 1 & & \\
\hline 98 & Pheles melanchroia (C. Felder \& R. Felder, 1865) & 1 & 1 & \\
\hline 99 & Pheles strigosa strigosa (Staudinger, 1876) & 1 & & \\
\hline 100 & Calephelis argyrodines (H.W. Bates,1866) & & 1 & 1 \\
\hline 101 & Calephelis azteca McAlpine, 1971 & 1 & & \\
\hline 102 & Calephelis browni McAlpine, 1972 & & 1 & \\
\hline 103 & Calephelis clenchi McAlpine, 1973 & & 1 & 1 \\
\hline 104 & Calephelis mexicana McAlpine, 1971 & 1 & & \\
\hline 105 & Calephelis huasteca McAlpine, 1971 & 1 & & \\
\hline 106 & Calephelis montezuma McAlpine, 1971 & 1 & & \\
\hline 107 & Calephelis acapulcoensis McAlpine, 1971 & 1 & & \\
\hline 108 & Calephelis yucatana McAlpine, 1971 & 1 & & \\
\hline 109 & Calephelis maya McAlpine, 1971 & & 1 & 1 \\
\hline 110 & Calephelis tikal Austin, 1993 & & 1 & 1 \\
\hline 111 & Caria domitianus vejento Clench, 1967 & 1 & & 1 \\
\hline
\end{tabular}


Cuadro 5. Continuación.

\begin{tabular}{|c|c|c|c|c|}
\hline \multirow[t]{2}{*}{ No. } & \multirow[t]{2}{*}{ PAPILIONOIDEA } & \multicolumn{3}{|c|}{ especies exclusivas } \\
\hline & & México & Guatemala & Belice \\
\hline 112 & Caria rhacotis (Godman \& Salvin, 1878) & 1 & & 1 \\
\hline 114 & Caria stillaticia Dyar, 1912 & 1 & & \\
\hline 115 & Baeotis sulphurea macularia (Boisduval, 1870) & 1 & & \\
\hline 118 & Mesene leucopus Godman \& Salvin, 1886 & & & 1 \\
\hline 119 & Esthemopsis alicia alicia (H.W. Bates, 1865) & & 1 & 1 \\
\hline 120 & Esthemopsis pherephatte (Godart, [1824]) & 1 & & \\
\hline 121 & Chimastrum argentea argentea (H.W. Bates, 1866) & 1 & & \\
\hline 125 & Anteros chrysoprasta roratus Godman \& Salvin, 1886 & 1 & 1 & \\
\hline 126 & Anteros formosus micon H. Druce, 1875 & 1 & & 1 \\
\hline 127 & Emesis fatimella nobilata Stichel, 1910 & 1 & & \\
\hline 128 & Emesis ocypore aethalia H.W. Bates, 1868 & 1 & 1 & \\
\hline 129 & Pseudonymphidia clearista (Butler, 1871) & 1 & & \\
\hline 130 & Pachythone gigas gigas Godman \& Salvin, 1878 & 1 & & \\
\hline 131 & Lemonias caliginea (Butler, 1867) & 1 & & \\
\hline 132 & Synargis nymphidioides ssp. $\mathrm{n}$. & 1 & 1 & 1 \\
\hline 133 & Synargis ethelinda (Hewitson, 1870) & & 1 & \\
\hline 139 & Calicosama lilina (Butler, 1870) & 1 & & \\
\hline 140 & Theope pedias Herrich-Schäffer, [1853] & 1 & 1 & 1 \\
\hline \multirow[t]{3}{*}{141} & Theope cratylus Godman \& Salvin, 1886 & 1 & 1 & \\
\hline & NYMPHALIDAE & & & \\
\hline & Ithomiinae & & & \\
\hline 142 & Melinaea lilis flavicans C.C. Hoffmann, 1924 & 1 & & \\
\hline 143 & Hypothyris euclea valora (Haensch, 1909) & 1 & 1 & 1 \\
\hline 144 & Dircenna dero euchytma (C. Felder \& R. Felder, 1865) & & 1 & 1 \\
\hline 145 & Dircenna dero occulta & & 1 & \\
\hline 146 & Dircenna dero ssp. n. & 1 & 1 & 1 \\
\hline 147 & Dircenna jemina chiriquensis Haensch, 1909 & & & 1 \\
\hline 148 & Pteronymia alcmena alcmena (Godman \& Salvin, 1877) & & 1 & \\
\hline \multirow[t]{2}{*}{149} & Godyris zavaleta sosunga (Reakirt, [1866]) & 1 & 1 & 1 \\
\hline & Morphinae & & & \\
\hline
\end{tabular}


Cuadro 5. Continuación.

\begin{tabular}{|c|c|c|c|c|}
\hline \multirow[t]{2}{*}{ No. } & \multirow[t]{2}{*}{ PAPILIONOIDEA } & \multicolumn{3}{|c|}{ especies exclusivas } \\
\hline & & México & Guatemala & Belice \\
\hline 150 & Antirrhea philoctetes casta H. Bates, 1865 & & & 1 \\
\hline 151 & Antirrhea philoctetes lindigii ((Fabricius 1793)) & & & 1 \\
\hline 152 & Morpho helenor octavia H.W. Bates, 1864 & 1 & & \\
\hline 153 & Morpho theseus justitiae Salvin \& Godman, 1868 & 1 & 1 & 1 \\
\hline 154 & Morpho theseus ssp. n. & 1 & & \\
\hline 155 & Caligo brasiliensis sulanus Fruhstorfer, 1904 & & 1 & 1 \\
\hline 156 & Caligo illioneus oberon Butler, 1870 & & & 1 \\
\hline 157 & Caligo oedipus fruhstorferi Stichel, 1904 & 1 & & \\
\hline 158 & Dynastor darius stygianus Butler, 1872 & 1 & & 1 \\
\hline 159 & Mimoblepia staudingeri mexicana (J. Maza \& R.G. Maza, 1989) & & & 1 \\
\hline 160 & Opsiphanes invirae relucens Fruhstorfer, 1907 & & 1 & \\
\hline \multirow[t]{2}{*}{161} & Narope testacea Godman \& Salvin, 1878 & 1 & & \\
\hline & Satyrinae & & & \\
\hline 162 & Cissia undina (Butler, 1870) & 1 & & \\
\hline 163 & Cyllopsis dospassosi L.D. Miller, 1974 & 1 & & \\
\hline 164 & Cyllopsis hedemanni tamaulipensis L.D. Miller, 1974 & 1 & & \\
\hline 165 & Cyllopsis pephredo (Godman, 1901) & & & 1 \\
\hline 166 & Cyllopsis suivalens escalantei L.D. Miller, 1974 & 1 & & \\
\hline 167 & Cyllopsis wellingi L. Miller, 1978 & & & 1 \\
\hline 168 & Magneuptychia alcinoe (C. Felder \& R. Felder, 1867) & 1 & & \\
\hline 169 & Megeuptychia antonoe (Cramer, 1775) & 1 & & \\
\hline 170 & Pseudodebis zimri (Butler, 1869) & 1 & 1 & 1 \\
\hline 171 & Taygetis inconspicua Draudt, 1931 & & & 1 \\
\hline 172 & Taygetis leuctra Butler, 1870 & & & 1 \\
\hline 173 & Taygetis uncinata Weymer, 1907 & 1 & & \\
\hline \multirow[t]{2}{*}{174} & Taygetis uzza Butler, 1869 & & & 1 \\
\hline & Charaxinae & & & \\
\hline 175 & Hypna clytemnestra mexicana A. Hall, 1917 & 1 & & \\
\hline 176 & Zaretis itys itys (Cramer, 1777) & & & 1 \\
\hline 177 & Fountainea ryphea ryphea (Cramer, 1775) & 1 & 1 & \\
\hline 178 & Memphis hedemanni (R. Felder, 1869) & 1 & 1 & 1 \\
\hline 179 & Memphis xenocles carolina W.P. Comstock, 1961 & 1 & & \\
\hline 180 & Prepona deiphile diaziana L.D. Miller \& J.Y. Miller, 1976 & & & 1 \\
\hline 181 & Prepona dexamenus medinai Beutelspacher, 1981 & 1 & 1 & 1 \\
\hline 182 & Agrias aedon rodriguezi Schaus, 1918 & 1 & & 1 \\
\hline 183 & Agrias amydon oaxacata Kruck, 1931 & 1 & & \\
\hline \multirow[t]{2}{*}{184} & Agrias amydon philatelica De Vries, 1980 & & & 1 \\
\hline & Biblidinae & & & \\
\hline 185 & Marpesia corita phiale (Godman \& Salvin, 1878) & 1 & 1 & \\
\hline 186 & Catonephele cortesi R.G. Maza, 1982 & 1 & & \\
\hline 187 & Eunica alpais excelsa Godman \& Salvin, 1877 & 1 & & \\
\hline
\end{tabular}


Cuadro 5. Continuación.

\begin{tabular}{|c|c|c|c|c|}
\hline \multirow[t]{2}{*}{ No. } & \multirow[t]{2}{*}{ PAPILIONOIDEA } & \multicolumn{3}{|c|}{ especies exclusivas } \\
\hline & & México & Guatemala & Belice \\
\hline 188 & Eunica malvina albida Jenkins, 1990 & 1 & & \\
\hline 190 & Nessaea aglaura aglaura (Doubleday, [1848]) & 1 & 1 & 1 \\
\hline 191 & Ectima erycinoides ssp. $\mathrm{n}$. & 1 & & \\
\hline 193 & Bolboneura sylphis veracruzana Draudt, 1931 & 1 & & \\
\hline 194 & Temenis laothoe laothoe (Cramer, 1777) & & 1 & \\
\hline 195 & Dynamine ate (Godman \& Salvin, 1883) & 1 & & \\
\hline 196 & Dynamine paulina thalassina (Boisduval, 1870) & & & 1 \\
\hline 197 & Dynamine theseus (C. Felder \& R. Felder, 1861) & 1 & 1 & 1 \\
\hline 201 & Callicore tolima guatemalena (H. W. Bates, 1866) & & 1 & 1 \\
\hline 202 & Adelpha ethelda ssp. $\mathrm{n}$. & 1 & & \\
\hline 203 & Adelpha erymanthis esperanza Balcazar \& Willmott, 2003 & & & 1 \\
\hline 204 & Adelpha iphiclus iphiclus (Linnaeus, 1758) & & 1 & 1 \\
\hline 205 & Adelpha malea fundania Fruhstorfer, 1915 & 1 & & \\
\hline \multirow[t]{2}{*}{206} & Adelpha nea sentia Godman \& Salvin, 1884 & & 1 & 1 \\
\hline & Nymphalinae & & & \\
\hline 207 & Baeotus beotus (Doubleday, [1849]) & 1 & & \\
\hline 214 & Anthanassa otanes otanes (Hewitson, 1864) & & 1 & \\
\hline 215 & Castilia ofella (Hewitson, [1864]) & 1 & & \\
\hline 216 & Ortilia orthia (Hewitson, 1864) & & & 1 \\
\hline \multirow[t]{2}{*}{217} & Tegosa claudina (Eschscholtz, 1821) & 1 & & \\
\hline & Heliconinae & & & \\
\hline \multirow[t]{3}{*}{218} & Actinote guatemalena guatemalena (H.W. Bates, 1864) & 1 & 1 & 1 \\
\hline & total de especies exclusivas a las SA en cada país & 144 & 82 & 92 \\
\hline & especies exclusivas a las SA presentes en un solo país & 85 & 23 & 37 \\
\hline
\end{tabular}

cada. En este trabajo Lycaenidae presentó mayor número de especies que Riodinidae, resultados que coinciden con los de la Selva Maya obtenidos por Salinas-Gutiérrez (2010). Los valores altos de riqueza de Theclinae (182) y Riodininae (133) son los esperados debido a la gran diversidad de especies vegetales que tienen las SA y a la gran especialización y diversificación de las familias a las que pertenecen estos taxones (Lycaenidae y Riodinidae, respectivamente) gracias a sus hábitos de alimentación larval monogenérica y monoespecífica con relación a varias plantas tropicales (Castillo-Guevara \& Rico-Gray, 2002; Contreras-Medina et al., 2003; Coto \& Saunders, 2001; 

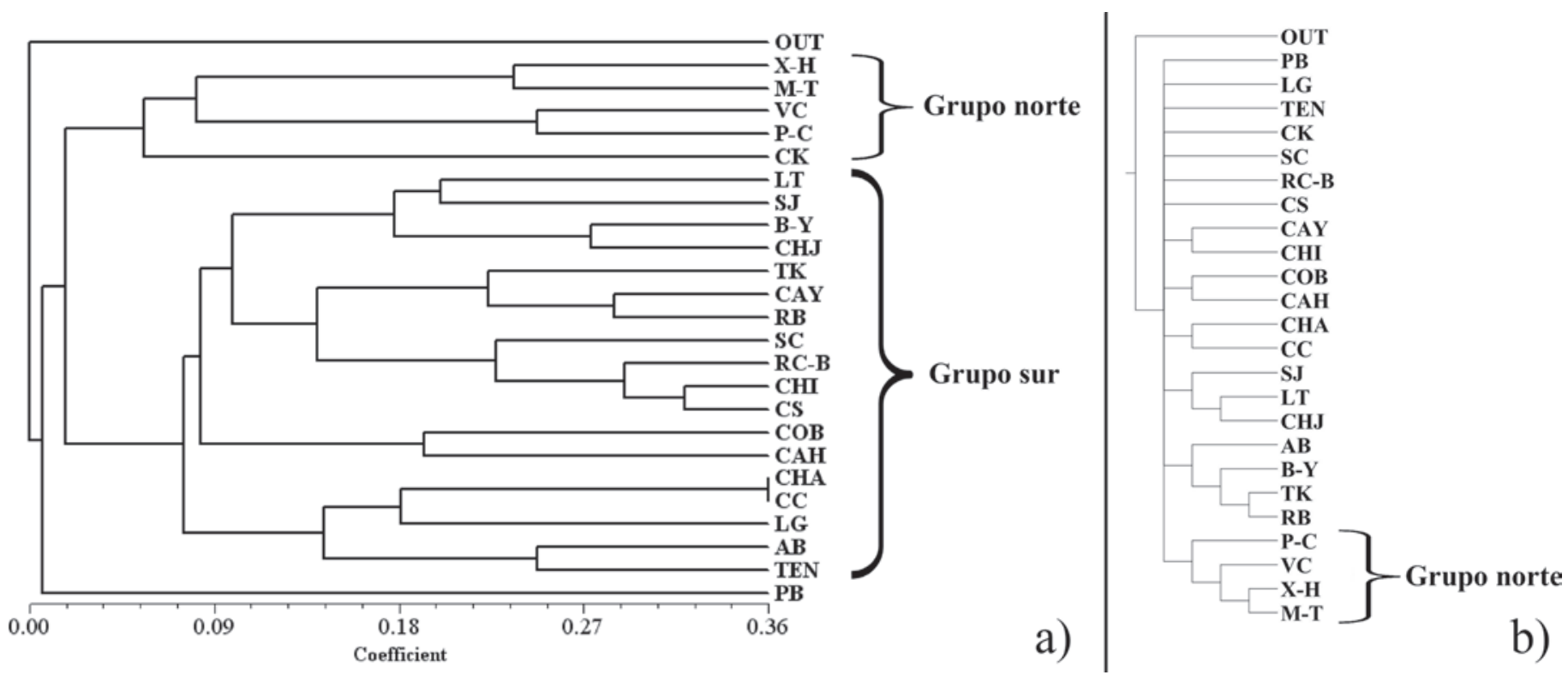

Figura 2. Dendrogramas que muestran la agrupación de los sitios con base en el Índice de Jaccard (a) y el PAE (b).

de la Maza, 1987; Maes, 2004; Vargas-Carrillo, 2011), así como hábitos mirmecófilos (de la Maza, 1987; Fürst \& Nash, 2010).

Algunos de los sitios más diversos (LT, 517; SJ, 365; CHJ, 364; X-H, 332; CAY, 329; PB, 318; TIK, 308) corresponden a las áreas con mayor tradición de estudio para cada país, mientras que los menos diversos (VC, 125; CAH, 90; CS, 71) están en las áreas menos estudiadas; adicionalmente, esta diferencia puede deberse a otros factores como la ubicación geográfica y al grado de perturbación de la vegetación en la zona y sus alrededores. Por ejemplo, los sitios X-H, PB y VC se localizan en el límite de distribución boreal de las SA en la PBGM y presentan parches de vegetación bien conservada rodeados por vegetación perturbada y zonas agrícolas, pero los dos primeros tienen una mayor tradición de estudio que VC; en el caso de TK respecto a CAH, TK cuenta con una vegetación más conservada y una mayor tradición de estudio que CAH. Para LT, SJ y CHJ su tradición de estudio es muy similar, aunque CHJ es el sitio de acceso más difícil por su ubicación geográfica y cuenta con una vegetación menos perturbada que los otros dos sitios, mientras que LT cuenta con un gradiente altitudinal mayor y se encuentra en una zona accesible rodeada por áreas sujetas a cambio de uso de suelo.

Al comparar el número de especies registradas en este trabajo con el total reportado para todo México (793 en SA/1190 en todo el país), Guatemala (405/749) y Belice
(422/545), se observa que más de la mitad se encuentran en las SA, lo que coincide con Dirzo (1990) y Wilson (1988) cuando afirman que las selvas tropicales cuentan con la mayor biodiversidad del planeta para diversos grupos de organismos, siendo al menos del 50\% (Dirzo, 1990; Wilson, 1988). El alto porcentaje de especies (77.43) que Belice presenta en las SA se debe a que gran parte de su territorio se encuentra cubierto por este tipo de vegetación; mientras que México (59.57) muestra un porcentaje superior al de Guatemala (54.07) debido a que tiene una superficie selvática más extensa.

El porcentaje elevado (45.78) de especies comunes (288 presentes en México, Guatemala y Belice) y potencialmente comunes a los tres países (76 presentes en México y Belice), puede explicarse si se considera que Belice, el norte de Guatemala y el sureste mexicano forman una unidad selvática continua dividida únicamente por las fronteras políticas (Salinas-Gutiérrez, 2010), que es la más grande de Mesoamérica y la segunda en importancia en América después de la Amazonia (Conservation International, 2004). Las especies reportadas sólo para Guatemala (26) y Belice (41), podrían ser encontradas en más de un país si se realizan más recolectas en los lugares poco estudiados de éstos y del sureste mexicano; las 271 especies presentes exclusivamente en México, se encuentran en la misma situación.

En este trabajo se agregó información para algunos de los 18 sitios de la Selva Maya analizados por Salinas- 
Gutiérrez (2010) y se incluyeron siete sitios adicionales, tres en Belice (RC-B, CHI y CS) y cuatro en México (MT, PB, VC y P-C); además, al revisar y actualizar las listas de los trabajos consultados en algunos casos se identificaron especies y subespecies distintas a las que menciona este autor. Por tales motivos, 46 de los 793 papilionoideos de las SA en la PBGM no están representados en el trabajo de la Selva Maya, y dado que en el presente análisis no se tomó en cuenta el sitio del Soconusco por ubicarse fuera de la PBGM, ni sus especies exclusivas, al comparar el total de Papilionoidea de las SA en la PBGM (793) con el total reportado para la Selva Maya (779) al final sólo se obtiene una diferencia de 14 especies. Se debe considerar que los sitios estudiados por Salinas-Gutiérrez (2010) fueron la base para este análisis ya que, debido al sesgo existente en el estudio de los bosques tropicales, son las áreas que cuentan con mayor información y accesibilidad, y a que su trabajo es el más completo para este tipo de vegetación en la región.

Las especies que se clasificaron como Generalistas (409) mostraron una presencia ecológica amplia, es decir, se encontraron en ambientes muy diversos, lo que permite suponer que tienen la capacidad de aprovechar distintos recursos en diversas condiciones, característica típica de una especie generalista o de nicho amplio (Rey-Benayas, 2009). Por otro lado, las de Presencia moderada (166) mostraron una incidencia limitada en otros tipos de vegetación además de las SA; lo que puede interpretarse como una aparición puntual breve en las SA si provienen de otro tipo de vegetación, o una aparición fuera de ellas si pertenecen a las SA y se desplazaron hacia el otro tipo de vegetación, característica que corresponde a las llamadas especies turistas (Halffter \& Moreno, 2005); estas suposiciones podrían respaldarse con un análisis de la abundancia de las especies registradas en cada sitio, dato que no está disponible en ninguno de los trabajos y que como consecuencia, no pudo analizarse en este estudio.

Bajo estas consideraciones, las 218 especies restantes se clasificaron como Exclusivas de las SA ya que cuentan con una mayor probabilidad de ser especies propias o características de esta vegetación, con una marcada dependencia a las condiciones ambientales de la SA (temperatura, humedad, especies vegetales, etc.), de tal manera que no sería posible su establecimiento y captura fuera de este tipo de vegetación. Entre las especies Exclusivas de las SA, 86 fueron registradas sólo en un sitio (Restringidas) por lo que podrían ser especies endémicas a un área particular dentro de la distribución total en este tipo de vegetación si se confirma que sólo están presentes en ese sitio. Es posible que algunas de estas especies tengan una distribución mayor y que incluso puedan dejar de ser consideradas Exclusivas de las SA, ya que si bien en este trabajo no fueron registradas fuera de las SA, algunas han sido reportadas por Llorente-Bousquets et al. (2006) en otras entidades mexicanas, sin precisar la vegetación en la que se encontraron.

Similitud y Análisis de parsimonia de endemismos. La agrupación de los sitios obtenida en los análisis refuerza la propuesta de separar a la PBGM en dos distritos, uno norte y otro sur a partir del extremo oriental del Eje Neovolcánico (Espinosa-Pérez \& Huidobro-Campos, 2005), que corresponde con la Sierra de Chiconquiaco (Aranda-Gómez et al., 2005; Cervantes-Zamora et al., 1990; Espinosa et al., 2008; Ferrari, 2000).

Una explicación histórica de la topología presentada por los dendrogramas y su relación con el área de la Sierra de Chiconquiaco se centra en el hecho de que a finales del Eoceno y principios del Oligoceno la vegetación tropical tenía su límite de distribución boreal en el sur y sureste de los actuales Estados Unidos de Norteamérica cerca de $\operatorname{los} 32^{\circ} \mathrm{N}$, y que después retrocedió hasta los $23^{\circ} \mathrm{N}$ como consecuencia del descenso de la temperatura iniciado en ese mismo periodo y que continuó con algunas interrupciones hasta el Mioceno superior; posteriormente, con la llegada del plioceno tardío y el inicio de las glaciaciones pleistocénicas, la distribución de la vegetación tropical se fragmentó y aisló por las condiciones húmedo-frías, seco-cálidas y seco-frías que reemplazaron a las cálidas y húmedas (González, 1998; Toledo,1976), aislamiento que se reforzó por el surgimiento del extremo oriental del Eje Neovolcánico durante el plio-pleistoceno.

Así, la agrupación de los sitios del conjunto norte en los dendrogramas, exceptuando a CK, corresponde a la porción de SA que fue aislada de las zonas tropicales del sur por el extremo oriental del Eje Neovolcánico (Sierra de Chiconquiaco), mientras que los sitios ubicados en el conjunto sur corresponden a la porción de selva que permaneció continua tras el surgimiento de la sierra durante el plio-pleistoceno (Aranda-Gómez et al., 2005). Este aislamiento y la reducción de la vegetación tropical durante las glaciaciones pleistocénicas, propiciaron que la composición de especies sea diferente en ambos lados de la sierra, ya que al terminar las glaciaciones los taxones tropicales se expandieron nuevamente a partir de los refugios pleistocénicos, todos ubicados al sur de Chiconquiaco (Toledo, 1976), excepto uno sugerido para Tamaulipas (González \& Hernández, 1998), las áreas del sur pudieron entrar en contacto nuevamente e intercambiar especies 
con áreas más australes, cálidas y húmedas, mientras que las del norte pudieron adoptar especies provenientes de latitudes boreales y zonas más frías y secas.

A pesar que existen especies exclusivas de las SA que se comparten entre los sitios de las dos vertientes de la sierra de Chiconquiaco (por ejemplo, Ocaria clenchi, Aubergina hicetas, Beotis sulphurea macularia y Castilia ofella), la agrupación norte en los dendrogramas se basa en la presencia de aquellas que en este trabajo sólo fueron encontradas al norte de esta sierra, como Calephelis montezuma, Ministrymon cleon y Texola elada ulrica; otras que se registraron únicamente en el sur y que definieron ese grupo fueron Arawacus togarna, Ziegleria hoffmani, Nessaea aglaura aglaura, Parides panares lycimenes y Actinote guatemalena guatemalena, sin embargo, los estudios de Llorente et al. (1997, 2006) y Luis et al. (2010), mencionan una distribución más amplia para algunas de estas especies.

El caso particular de la inclusión de CK con los sitios ubicados al norte de la sierra, inicialmente se interpreta como una agrupación de las SA más secas de la provincia, los sitios del norte como consecuencia de su mayor latitud y CK por la situación orográfica particular de la Península de Yucatán; aunque al observar la lista de especies existe la posibilidad de que se trate de una distribución disyunta de aquellas que lograron establecerse en ambos lados de la sierra. CK ubicado al sur de Chiconquiaco fue asociado con los sitios del norte debido a que compartió las especies Strymon mulucha (X-H y P-C), Junonia evarete ssp. n. (X-H) y Pseudonymphidia clearista (X-H y M-T).

PB presenta vegetación fuertemente perturbada, es el sitio que se interna más y a mayor altitud dentro de la Sierra Madre Oriental, y el único próximo al punto de contacto entre tres subprovincias fisiográficas (Llanuras y sierras de Querétaro e Hidalgo, Lagos y volcanes de Anáhuac y el Carso Huasteco) de acuerdo con Cervantes-Zamora et al. (1990), factores que favorecen el flujo de especies turistas hacia las SA y viceversa, las cuales fueron excluidas del estudio al aplicar los criterios del método de selección de los taxones; en consecuencia, PB comparte únicamente tres especies con el resto de los sitios, Nicolaea velina (LT, SJ y CHJ), $N$. heraldica (LT y CC) y Gargina gargophia (LT). Un análisis con datos de abundancia permitiría una selección más adecuada de las especies características de las SA, y con ello posiblemente se obtendría un resultado que mostrara la pertenencia de $\mathrm{PB}$ a alguna de las agrupaciones.

Análisis en los que se describen e interpretan las relaciones entre los distintos sitios con SA en la provincia
(Maya-Martínez et al., 2005; Salinas-Gutiérrez, 2010; Salinas-Gutiérrez et al., 2004, 2006) muestran que CHJ, SJ, LT y SLP (San Luis Potosí, equivalente en este trabajo a $\mathrm{X}-\mathrm{H}$ ) forman un grupo estable (obtenido mediante análisis de parsimonia y similitud). Sus conclusiones generales sugieren que el bosque tropical perennifolio formaba un continuo que se extendía sobre la vertiente atlántica de los tres países, mostrando así la relación existente entre las distintas áreas con esta vegetación. Los resultados del presente trabajo confirmaron la consistencia de la agrupación de CHJ, SJ y LT, mientras que la separación de $\mathrm{X}$-H demuestra que a pesar de formar parte de la misma unidad de selvas de la provincia, pertenece a un grupo distinto; entre otras causas, esta separación se define por la diferencia en la composición de especies generada por el aislamiento del norte respecto al sur ocasionado por la Sierra de Chiconquiaco.

\section{CONCLUSIONES}

La diversidad de Papilionoidea en las selvas altas (SA) de la Provincia Biogeográfica del Golfo de México (PBGM) es de 793 especies; es el valor máximo que se ha referido para este tipo de vegetación incluso comparado con la diversidad registrada en la Selva Maya, la cual se extiende en otras provincias biogeográficas. Por primera vez se ci$\tan 46$ especies y subespecies de papilionoideos para las SA.

El patrón de riqueza por familia de los papilionoideos de la PBGM es el mismo que se ha encontrado en otras áreas neotropicales para papiliónidos, piéridos y ninfálidos, aunque en cuanto a licénidos y riodínidos más bien coincide con las proporciones detectadas en la Selva Maya; las subfamilias con mayor riqueza fueron Theclinae (182 especies) y Riodininae (133).

Aproximadamente la mitad de las especies de estas selvas se compartieron o son potencialmente comunes a Belice, Guatemala y México, aunque este último contiene la gran mayoría de los papilionoideos exclusivos a un país.

Se confirmó la gran diversidad específica que albergan los bosques tropicales, y en particular las SA ya que más de la mitad de los papilionoideos presentes en los países analizados se encontraron en esta vegetación (México $59.57 \%$, Guatemala 54.07\% y Belice $77.43 \%$ ), concentrados principalmente en un privilegiado grupo de sitios ubicados a lo largo de esta provincia entre los que destaca Los Tuxtlas como el más diverso (517 especies). 
Se propone un conjunto constituido por 218 especies características de las SA para el estudio y conservación de este tipo de vegetación, 86 de ellas distribuidas exclusivamente en un sólo sitio dentro de la provincia del Golfo de México.

Finalmente, este análisis coincide con la propuesta de separación de la Provincia Biogeográfica del Golfo de México en dos distritos a partir de la Sierra de Chiconquiaco: el Norte (X-H, M-T, VC, P-C y CK -aunque éste último geográficamente se encuentra en el sur) y el Sur (LT, SJ, B-Y, CHJ, TK, CAY, RB, SC, RC-B, CHI, CS, COB, CAH, CHA, CC, LG, AB y TEN).

AGRADECIMIENTOS. A la Carrera de Biología de la FES Zaragoza, UNAM. Al Museo de Zoología de la Facultad de Ciencias, en especial al M. en C. Armando Luis-Martínez por la facilidad otorgada para consultar la base de datos MARIPOSA.

\section{LITERATURA CITADA}

Aranda-Gómez, J.J., Luhr, J.F., Housh, T. B., Valdez-Moreno, G. \& Chávez-Cabello, G. (2005). El volcanismo tipo intraplaca del Cenozoico tardío en el centro y norte de México: una revisión. Boletín de la Sociedad Geológica Mexicana, 57, 187-225.

Austin, G. T., Haddad, N. M., Méndez, C., Sisk, T. D., Murphy, D. D., Launer A. E. \& Ehrlich, P. R. (1996). Annoted checklist of the Tikal National Park area of Guatemala. Tropical Lepidoptera, 7, 21-37.

Barrios, M. V., Hernández, S. M., Pardo, P. D., Herrera, F., Méndez, C., Orellana, R., Castillo P. \& Burgos, C. L. (2007). Exploración del potencial de cultivos alternativos perennes de alto valor (Theobroma cacao L.), aplicados en aéreas selectas para la restauración de las selvas lluviosas fragmentadas en Cobán y Cahabón, Alta Verapaz. Centro de Datos para la Conservación (CDC) del Centro de Estudios Conservacionistas (CECON) y Escuela de Biología (FAC., CCQQ y F., USAC), Guatemala, 116 p.

Castillo-Guevara, C. \& Rico-Gray, V. (2002). Is cycasin in Eumaeus minyas (Lepidoptera: Lycaenidae) a predator deterrent? Interciencia, 27, 465-470.

CCAD. (2015a). Comisión Centroamericana de Ambiente y Desarrollo. Coberturas vectoriales en Mesoamérica. Curvas de nivel de Belice. http://www.sica.int/ccad/mapas.aspx (consultado: 15 de mayo de 2015).

CCAD. (2015b). Comisión Centroamericana de Ambiente y Desarrollo. Coberturas vectoriales en Mesoamérica. Curvas de nivel de Guatemala. http://www.sica.int/ccad/mapas.aspx (consultado: 15 de mayo de 2015).

CCAD. (2015c). Comisión Centroamericana de Ambiente y Desarrollo. Coberturas vectoriales en Mesoamérica. Ecosistemas de Belice. http://www.sica.int/ccad/mapas.aspx (consultado: 15 de mayo de 2015).

CCAD. (2015d). Comisión Centroamericana de Ambiente y Desarrollo. Coberturas vectoriales en Mesoamérica. Ecosistemas de
Guatemala. http://www.sica.int/ccad/mapas.aspx (consultado: 15 de mayo de 2015).

Cervantes, F. A., Castro-Campillo, A. \& Ramírez-Pulido, J. (1994). Mamíferos Terrestres Nativos de México. Anales del Instituto de Biología, UNAM, Serie de Zoología, 65, 177-190.

Cervantes-Zamora, Y., Cornejo-Olguín, S. L., Lucero-Márquez, R., Espinoza-Rodríguez, J. M., Miranda-Viquez, E. \& Pineda-Velázquez, A. (1990). Provincias Fisiográficas de México. Extraído de Clasificación de Regiones Naturales de México II, IV.10.2. Atlas Nacional de México. Vol. II. Escala 1:4 000000. Instituto de Geografía, UNAM. México. http://www.conabio.gob. mx/informacion/gis/ (consultado: 15 de mayo de 2015).

Challenger, A. \& Soberón, J. (2008). Los ecosistemas terrestres. Pp. 87-108. In: J. Soberón, G. Halffter \& J. Llorente-Bousquets (Eds.). Capital natural de México. vol. I: Conocimiento actual de la biodiversidad. CONABIO (Comisión Nacional para el Conocimiento y Uso de la Biodiversidad), México, D. F.

Cherrington, E. A., Ek, E., Cho, P., Howell, B. F., Hernández, B. E., Anderson, E. R., Flores, A. I., García, B. C., Sempris E. \& Irwin, D. E. (2010). Forest Cover and Deforestation in Belize: 1980-2010. Sistema regional de visualización y monitoreo para Mesoamérica (SERVIR), Centro del Agua del Trópico Húmedo para América Latina y el Caribe (CATHALAC). Panamá, Panamá. $42 \mathrm{p}$.

CNINLEPIDOPTERA. (2010). Colección Nacional de Insectos Lepidoptera. Portal UNIBIO (Unidad de Informática para la Biodiversidad). Instituto de Biología, UNAM. http://www.unibio.unam. mx (Consultado: 15 de septiembre de 2010).

CONABIO. (1998). Comisión Nacional para el Conocimiento y Uso de la Biodiversidad. Curvas de nivel para la República Mexicana. Escala 1:250000. Extraído del Modelo Digital del Terreno. Instituto Nacional de Estadística, Geografía e Informática (INEGI). México. http://www.conabio.gob.mx/informacion/gis/ (consultado: 15 de mayo de 2015).

CONABIO. (1999). Comisión Nacional para el Conocimiento y Uso de la Biodiversidad. Uso de suelo y vegetación modificado por CONABIO. Escala 1:1 000000. México. http://www.conabio.gob. mx/informacion/gis/ (consultado: 15 de mayo de 2015).

Conservation International. (2004). Perfil de ecosistema. Región norte del hotspot de biodiversidad de Mesoamérica: Belice, Guatemala, México. Critical Ecosystem Partnership Fund, 64 p.

Contreras-Medina, R., Ruiz-Jiménez, C. A. \& Luna-Vega, I. (2003). Caterpillars of Eumaeus childrenae (Lepidoptera; Lycaenidae) feeding on two species of cycads (Zamiaceae) in the Huasteca region, Mexico. Revista de Biología Tropical/International Journal of Tropical Biology and Conservation, 51, 201-204.

Coto, D. \& Saunders, J. L. (2001). Insectos plaga de la guanábana (Annona muricata) en Costa Rica. Manejo Integrado de Plagas, 61, 60-68.

Dirzo, R. 1990. La biodiversidad como crisis ecológica actual ¿Qué sabemos? Ciencias, Número especial 4, 48-55.

Escalante, T. \& Morrone, J. J. (2003). ¿Para qué sirve el análisis de parsimonia de endemismos? Pp. 167-172. In: J. J. Morrone \& J. Llorente Bousquets (Eds.). Una perspectiva latinoamericana de la biogeografía. Las Prensas de Ciencias, UNAM. México, D. F.

Espinosa, D., Ocegueda, S., Aguilar, C., Flores, O., Llorente-Bousquets, J. \& Vázquez, B. (2008). El conocimiento biogeográfico 
de las especies y su regionalización natural. Pp. 33-65. In: J. Soberón, G. Halffter \& J. Llorente-Bousquets (Eds.). Capital natural de México. vol. I: Conocimiento actual de la biodiversidad. CONABIO (Comisión Nacional para el Conocimiento y Uso de la Biodiversidad). México, D. F.

Espinosa-Pérez, H. \& Huidobro-Campos, L. (2005). Ictiogeografía de los peces dulceacuícolas de la vertiente del Golfo de México. Pp. 295-318. In: J. Llorente-Bousquets \& J. J. Morrone (Eds.). Regionalización Biogeográfica en Iberoamérica y tópicos afines: Primeras Jornadas Biogeográficas de la Red Iberoamericana de Biogeografía y Entomología Sistemática (RIBES XII.I-CYTED). Las Prensas de Ciencias. UNAM. México.

Ferrari, L. (2000). Avances en el conocimiento de la Faja Volcánica Transmexicana durante la última década. Boletín de la Sociedad Geológica Mexicana, 53, 84-92.

Fürst, M. A. \& Nash, D. R. (2010). Host ant independent oviposition in the parasitic butterfly Maculinea alcon. Biology Letters, 6, 174176.

Gibbs, A. E. 1912. Butterflies from British Honduras and Guatemala. Proceedings of the Entomological Society of London, 2, 45-48.

Gibbs, A. E. (1914). An expedition to the Sarstoon River. Proceedings of the Entomological Society of London, 1: 20-23.

Godman, F. D. \& Salvin, O. (1879-1901). Biologia Centrali Americana. Zoología, Insecta, Lepidoptera, Rhopalocera. Volúmenes I-III. In: Electronic Biologia Centrali-Americana. http://www.sil. si.edu/digitalcollections/bca/explore.cfm (consultado: 15 de mayo de 2015).

González, F. \& Hernández, G. 1998. Las dolinas de Tamaulipas. Ciencias, 50, 56-58.

González, F. (1998). La vegetación de México y su historia. Ciencias, 52, 58-65.

González-Martínez, M. de L. (1996). Listado faunístico de los Papilionoidea del estado de Puebla, con especial referencia a la zona de Barranca de Patla. Tesis de Licenciatura en Biología. Facultad de Ciencias. UNAM. México. 112 p.

González-Valdivia, N., Pozo, C., Ochoa-Gaona, S., Gordon, F. B., Cambranis, E., Lara, O., Pérez-Hernández, I., Ponce-Mendoza, A. \& Kampichler, C. (2016). Nymphalidae frugívoras (Lepidoptera: Papilionoidea) asociadas a un ecomosaico agropecuario y de bosque tropical lluvioso en un paisaje del sureste de México. Revista Mexicana de Biodiversidad, 87, 451-464.

Halffter, G. \& Moreno, C. E. (2005). Significado biológico de las diversidades Alfa, Beta y Gamma. Pp. 5-18. In: G. Halffter, J. Soberón, P. Koleff \& A. Melic (Eds.). Sobre Diversidad Biológica: el Significado de las Diversidades Alfa, Beta y Gamma. m3m: Monografías Tercer Milenio, vol. 4, SEA (Sociedad Entomológica Aragonesa). Zaragoza, España.

Hernández-Baz, F., Luna, P. D., Castro, B. G., Bradleigh, V. S. \& González, G. (2016). Species richness of butterflies (Papilionoidea) at Natura Park, Xalapa, Veracruz, Mexico. Southwestern Entomologist, 41, 417-43

Jaccard, P. (1908). Nouvelles recherches sur la distribution florale. Bulletin de la Société Vaudoise des Sciences Naturelles, 44, 223270.

Koleff, P. (2005). Conceptos y medidas de la diversidad beta. Pp. 1940. In: G. Halffter, J. Soberón, P. Koleff \& A. Melic (Eds.). Sobre Diversidad Biológica: el Significado de las Diversidades Alfa,
Beta y Gamma. m3m: Monografías Tercer Milenio, vol. 4, SEA (Sociedad Entomológica Aragonesa). Zaragoza, España.

Lamas, G. (2008). La sistemática sobre mariposas (Lepidoptera: Hesperioidea y Papilionoidea) en el mundo: Estado actual y perspectivas futuras. Pp. 57-70. In: J. Llorente-Bousquets \& A. Lanteri (Eds.). Contribuciones taxonómicas en ordenes de insectos hiperdiversos. Las Prensas de Ciencias. UNAM. México, D. F.

Llorente-Bousquets, J., Luis-Martínez, A. \& Vargas-Fernández, I. (2006). Apéndice general de Papilionoidea: Lista sistemática, distribución estatal y provincias Biogeográficas. Pp. 945-1009. In: J. J. Morrone \& J. Llorente-Bousquets (Eds.). Componentes bióticos principales de la entomofauna mexicana. Las Prensas de Ciencias. UNAM. México, D. F.

Llorente, J., Oñate, L., Luis, A. \& Vargas, I. (1997). Papilionidae y Pieridae de México: distribución geográfica e ilustración. México, D.F. Comisión Nacional para el Conocimiento y Uso de la Biodiversidad y Facultad de Ciencias, Universidad Nacional Autónoma de México.

Llorente-Bousquets, J., Vargas-Fernández, I., Luis-Martínez, A., Trujano-Ortega, M., Hernández-Mejía, B. C. \& Warren, A. D. (2014). Biodiversidad de Lepidoptera en México. Revista Mexicana de Biodiversidad, Suplemento. 85, 353-371.

Luis-Martínez, A., Llorente-Bousquets, J. \& Vargas-Fernández, I. (2005). Una megabase de datos de mariposas y la regionalización biogeográfica de México. Pp. 269-294. In: J. Llorente \& J. J. Morrone (Eds.). Regionalización geográfica en Iberoamérica y tópicos afines: Primeras Jornadas Biogeográficas de la Red Iberoamericana de Biogeografía y Entomología Sistemática (RIBES XII.I-CYTED). UNAM. México.

Luis-Martínez, A., Llorente-Bousquets, J., Warren, A. D. \& Vargas-Fernández, I. (2004). Lepidópteros: papilionoideos y hesperioideos. Pp. 335-355. In: A. J. García-Mendoza, M. J. Ordoñez \& M. Briones-Salas (Eds.). Biodiversidad de Oaxaca. Instituto de Biología. UNAM, Fondo Oaxaqueño para la Conservación de la Naturaleza, World Wildlife Fund. México.

Luis-Martínez, A., Vargas-Fernández I. \& Llorente-Bousquets. J. (1995). Síntesis de los Papilionoidea (Lepidoptera: Rhopalocera) del estado de Veracruz. Folia Entomológica, 93, 91-133.

Luis, A., Llorente, J., Vargas, I. \& Pozo, C. (2010). Nymphalidae de México III (Nymphalinae). Distribución geográfica e ilustración. México, D.F: Facultad de Ciencias, Universidad Nacional Autónoma de México.

Luis-Martínez, M. A., Llorente-Bousquets, J. E., Vargas-Fernández, I. \& Hernández-Baz, F. (2011). Mariposas diurnas Papilionoidea y Hesperoidea (Insecta: Lepidoptera). Pp. 339-354. In: A. Cruz A. (Ed.). La biodiversidad de Veracruz: Estudio de Estado. Comisión Nacional para el Conocimiento y Uso de la Biodiversidad. Gobierno del Estado de Veracruz, Universidad Veracruzana, Instituto de Ecología, A.C. México.

Luis-Martínez, M. A., Vargas-Fernández, I. \& Llorente-Bousquets, J. E. (1991). Lepidopterofauna de Oaxaca I. Distribución y Fenología de los Papilionoidea de la Sierra de Juárez. Publicaciones Especiales del Museo de Zoología No. 3. Facultad de Ciencias. UNAM. México. 119 p.

Maes, J. M. (2004). Insectos asociados a algunos cultivos tropicales en el atlántico de Nicaragua. Parte XIII. Piña (Ananas comosus, 
Bromeliaceae). Revista Nicaragüense de Entomología, 64 (suplemento 1): 1-67.

Magurran, A. E. (1988). Ecological diversity and its measurement. Princeton University Press. Princeton, New Jersey. 179 p.

Martínez, G. L. (1994). Inventario de la división Rhopalocera (Lepidoptera: Frenatae) del parque estatal de Agua Blanca, Tabasco. Tesis de Licenciatura, División Académica de Ciencias Biológicas, Universidad Juárez Autónoma de Tabasco. 54 p.

Maya-Martínez, A., Pozo C. \& May-Uc, E. (2005). Las mariposas (Rhopalocera: Papilionidae, Pieridae y Nymphalidae) de la selva alta subperennifolia de la región de Calakmul, México, con nuevos registros. Folia Entomológica Mexicana, 44, 123-143.

Maza, J. de la \& Maza, R. de la. (1985a). La fauna de mariposas de Boca de Chajul, Chiapas, México, (Rhopalocera). Parte I. Revista de la Sociedad Mexicana de Lepidopterología, 9, 23-44.

Maza, J. de la \& Maza, R. de la. (1985b). La fauna de mariposas de Boca de Chajul, Chiapas, México, (Rhopalocera). Parte II. Revista de la Sociedad Mexicana de Lepidopterología, 10, 1-24.

Maza, R. de la. (1987). Mariposas Mexicanas. Guía para su colecta y determinación. Fondo de Cultura Económica. México, D. F. 301 p.

Meerman, J. C. \& Clabaugh, J. (2012). BERDS (Biodiversity and Environmental Resource Data System of Belize). http://www.biodiversity.bz (consultado: 15 de mayo de 2015).

Meerman, J. C. (1999). Lepidoptera of Belize. 1. Catalog of butterflies (Lepidoptera: Papilionoidea). Tropical Lepidoptera, 10 (supl. 1), 7-32.

Michán, L., Llorente-Bousquets, J., Luis-Martínez, A. \& JimenaCastro, D. (2005). Breve historia de la taxonomía de Lepidoptera en México durante el siglo XX. Revista de la Academia Colombiana de Ciencias Exactas, Físicas y Naturales, 29, 101-132.

Microsoft. (2013). Microsof Office Professional Plus Excel for Windows. Microsoft Corporation.

Moreno, C. E. (2001). Métodos para medir la biodiversidad. M\&TManuales y Tesis SEA (Sociedad Entomológica Aragonesa). Vol. 1. Zaragoza, España. 84 p.

Morrone, J. J. (1994a). Distributional patterns of species of Rhytirrhinini (Coleoptera: Curculionidae) and the historical relationships of the Andean Provinces. Global Ecology and Biogeography Letters, 4, 188-194.

Morrone, J. J. (1994b). On the identification of areas of endemism. Systematic Biology. 43, 438-441.

Morrone, J. J. (2001). Biogeografía de América Latina y el Caribe. M\&T-Manuales y Tesis SEA (Sociedad Entomológica Aragonesa). Vol. 3. Zaragoza, España. 150 p.

Morrone, J. J. (2004). Panbiogeografía, componentes bióticos y zonas de transición. Revista Brasileira de Entomología, 48, 149-162.

Morrone, J. J. (2014). Parsimony analysis of endemicity (PAE) revisited. Journal of Biogeography, 41, 842-854.

Morrone, J. J., Espinosa-Organista, D. \& Llorente-Bousquets, J. (2002). Mexican Biogeografic Provinces: Preliminary Scheme, General Characterizations, and Synonymies. Acta Zoológica Mexicana (n.s.), 85, 83-108.

Morrone, J. J., Espinosa-Organista, D., Aguilar-Zúñiga, C. \& Llorente-Bousquets, J. (1999). Preliminary classification of the Mexican Biogeographic Provinces: A Parsimony Analysis of Endemicity based on plant, insect and bird taxa. The Southwestern Naturalist, 44, 507-514.
Nieukerken, E. van, Kaila, L., Kitching, I., Kristensen, N., Lees, D., Minet, J., Mitter, C., Mutanen, M., Regier, J., Simonsen, T., Wahlberg, N., Yen, S., Zahiri, R., Adamski, D., Baixeras, J., Bartsch, D., Bengtsson, B., Brown, J., Bucheli, S., Davis, D., De Prins, J., De Prins, W., Epstein, M., Gentili-Poole, P., Gielis, C., Hättenschwiler, P., Hausmann, A., Holloway, J., Kallies, A., Karsholt, O., Kawahara, A., Koster, S., Kozlov, M., Lafontaine, D., Lamas, G., Landry, J., Lee, S., Nuss,M., Park, K., Penz, C., Rota, J., Schintlmeister, A., Schmidt, C., Sohn, J., Solis, A., Tarmann, G., Warren, A., Weller, S., Yakovlev, R., Zolotuhin, V. \& Zwick, A. (2011). Order Lepidoptera Linnaeus, 1758. Zootaxa, 3148, 211-221.

Nixon, K. C. (1999). Winclada, Versión 1.00.08. Publicado por el autor. Ithaca, New York.

Pozo, C., Luis-Martínez, A., Uc-Tescum, S., Salas-Suárez, N. \& Maya-Martínez, A. (2003). Butterflies (Papilionoidea and Hesperioidea) of Calakmul, Campeche, México. The Southwestern Naturalist, 48, 505-525.

Raguso, R. A. \& Llorente-Bousquets, J. (1990). The Butterflies (Lepidoptera) of the Tuxtlas Mts., Veracruz, Mexico, Revisited: Species-Richness and Habitat Disturbance. Journal of Research on the Lepidoptera, 29,105-133.

Raguso, R. A. \& Llorente, J. (1997). Papilionoidea. Pp. 257-291. In: E. González, R. Dirzo \& R. Vogt (Eds.). Historia Natural de los Tuxtlas. Instituto de Biología. UNAM.

Rey-Benayas, J. M. (2009). La rareza de las especies. Investigación y Ciencia, 392, 62-69.

Rohlf, F. (2004). NTSYSpc: Numerical taxonomy and multivariate analysis system. Version 2.11T. Exeter Software, New York.

Salinas-Gutiérrez, J. L. (2010). Biogeografía de las mariposas (Rhopalocera: Papilionoidea) de la Selva Maya de México, Guatemala y Belice. Tesis de Doctorado en Ciencias en Ecología y Desarrollo Sustentable. ECOSUR. Chetumal, Quintana Roo, México. 208 p.

Salinas-Gutiérrez, J. L., Llorente-Busquets, J. E., Méndez, C., Barrios, M. \& Pozo, C. (2012). Introducción a los Papilionoidea (Papilionidae, Pieridae, Lycaenidae, Riodinidae y Nymphalidae) de Guatemala. Pp. 155-171. In: E. B. Cano \& J. C. Schuster (Eds.). Biodiversidad de Guatemala. Volumen II. Universidad del Valle de Guatemala. Guatemala.

Salinas-Gutiérrez, J. L., Luis-Martínez, A. \& Llorente-Busquets, J. (2004). Papilionoidea of the Evergreen Tropical Forests of México. Journal of the Lepidopterists' Society, 58, 125142.

Salinas-Gutiérrez, J. L., Méndez, C., Barrios, M., Pozo, C. \& Llorente-Bousquets, J. (2009). Hacia una síntesis de los Papilionoidea (Insecta: Lepidoptera) de Guatemala con una reseña histórica. Caldasia, 31, 407-440.

Salinas-Gutiérrez, J. L., Pozo, C. \& Luis-Martínez, A. (2006). Relaciones biogeográficas de Papilionidae, Pieridae y Nymphalidae (Rhopalocera: Papilionoidea) en el bosque tropical perennifolio de México. Entomotropica, 21, 145-152.

Southwood, T. R. E. \& Henderson, P. A. (2000). Ecological Methods. 3th edition. Blackwell Science Ltd. Oxford. 575 p.

Toledo, V. M. (1976). Los cambios climáticos del pleistoceno y sus efectos sobre la vegetación tropical cálida y húmeda de México. Tesis de Maestría en Ciencias (Biología). Facultad de Ciencias. UNAM. México. 73 p. 
Flores-Contreras y Luna-Reyes: Papilionoidea de las selvas altas en el Golfo de México

Vargas-Carrillo, E. (2011). Guía para la identificación y manejo integrado de plagas en piña. REPCar (Reduciendo el Escurrimiento de Plaguicidas al Mar Caribe), Proagroin. Costa Rica. 29 p.

Villegas, I. S. (1998). Inventario de los Papilionoidea (Insecta: Lepidoptera) del Monumento Natural Cerro del Coconá, Teapa, Tabasco y algunos aspectos sobre su fenología. Tesis de Licenciatura, División Académica de Ciencias Biológicas, Universidad Juárez Autónoma de Tabasco. 87 p.

Warren, A. D., Davis, K. J., Stangeland, E. M., Pelham, J. P. \& Grishin, N. V. (2013). Illustrated Lists of American Butterflies. http://www.butterfliesofamerica.com (consultado: 15 de mayo de
2015).

Welling, E. C. (1973). The 1972 field season summary: corrections and additions. Northern Neotropics. News of the Lepidopterists' Society, 3, 5-6.

Welling, E. C. (1975). The 1974 field season summary. Northern Neotropics. News of the Lepidopterists' Society, 2/3, 17.

Welling, E. C. (1977). The annual Field Summary for 1977. Northern Neotropics. News of the Lepidopterists' Society, 2, 19-21.

Wilson, E. O. (1988). The current state of biological diversity. Pp. 318. In: E. O. Wilson (Ed.). Biodiversity. National Academy Press. Washington, D. C. 